\title{
Aspectos epidemiológicos e clínicos da intoxicação por Pithomyces chartarum em ovinos da raça Santa Inês, no município de Gravatá - PE
}

\section{Epidemiological and clinical aspects of Pithomyces chartarum intoxication in Santa Inês ovine in Gravatá City - PE}

\author{
Pierre Castro Soares, ${ }^{\star}$ Rinaldo Aparecido Mota, ${ }^{* *}$ Miriam Nogueira Teixeira, ${ }^{*}$ Néria Vânia Marcos dos Santos
}

\begin{abstract}
Resumo
Registra-se ocorrência de intoxicação por Pithomyces chartarum em ovinos da raça Santa Inês, em pastagem de Brachiaria decumbens, no município de Gravatá-PE, com os seguintes achados clínico-patológicos: anorexia, apatia, icterícia, edema palpebral e de orelhas, opacidade de córnea, exsudação purulenta ocular, dermatite na face interna da orelha, febre, desidratação; fígado aumentado e sensível, redução do tempo de coagulação, decúbito e morte; anemia normocítica e hipocrômica, inversão na relação neutrófilo-linfócito, aumento de Aspartato Amino-Transferase, Gama GT, elevação das Bilirrubinas Total, Direta e Indireta, além de hemoglobinúria. À necropsia observou-se icterícia generalizada, fígado amarelado e aumentado; congestão intestinal; baço discretamente aumentado e congesto. Conídeos do fungo $P$. chartarum foram identificados na pastagem e cujas toxinas foram responsáveis pelas lesões de fotossensibilização hepatógena em ovinos com pelagem e pêlos pretos.
\end{abstract}

Palavras-chave: Pithomyces chartarum; Brachiaria decumbens; fotossensibilização; ovinos.

\begin{abstract}
Intoxication occurence by Pithomyces chartarum is registred in ovine herds of "Santa Inês" breed in pasture of Brachiaria decumbens in Gravatá City - PE, with the following clinical-pathological finds: anorexia, apathy, jaundice, palpebral and auricular edema, cornea opacity, ocular purulent exudation, inner auricular dermatitis, fever, dehydration, liver region increased and sensible, coagulation time reduction, decubitus and death; hypochromic and normochromic anaemia, invertion of the relation neutrocyte-lymphocyte; increase of Aspartate-aminotransferase and Gamma Glutamyl Transferase, total conjuged and nonconjuged bilirubin rise, as well as hemoglobinuria. At the necropsy was observed generalized icterus and increased liver; intestinal congestion; spleen discreetely enlarged and congested. Conidia of the aforesaid fungus were identified, which toxin were responsable for the heatogenous photosensitization lesions in ovine with black coat and skin.
\end{abstract}

Keywords: Pithomyces chartarum; Brachiaria decumbens; photosentitization; ovines.

\section{Introdução}

A ocorrência de fotossensibilização hepatógena em ruminantes mantidos em pastagem de Brachiaria decumbens tem sido constante no Brasil, sendo responsável por significativas perdas econômicas com morte de grande número de animais.

Dentre as espécies animais que, sob condições naturais, tem sido observada a fotossensibilização, estão a bovina, a bubalina e a ovina (Tokarnia et al., 1979 ; Schenk, Schenk, 1983).

O processo de fotossensibilização ocorre em animais mantidos em pastagens contaminadas por Pithomyces chartarum (Bert, Curt ; M.B. Ellis, fungo pertencente à família Dematiaceae, considerado saprófita para vegetais
(Döbereiner et al., 1976 ; Schenk, Schenk, 1983 ; Purchio et al., 1988). Tal processo tem sido relatado mais freqüentemente na Nova Zelândia (DiMenna, Barley, 1973 ; Richard, 1973), Austrália (Hore, 1960), África do Sul (Coetzer et al., 1983), Estados Unidos (Muchiri et al., 1980 ; Bagley, 1983 ; Hansen et al., 1994). No Brasil, os primeiros relatos foram feitos no Mato Grosso (Camargo et al., 1976 ; Döbereiner et al., 1976), Goiás (Nobre, Andrade, 1976), São Paulo (Camargo et al., 1976), Pará (Camargo et al., 1976) e Mato Grosso do Sul (Fagliari et al., 1991a).

Disfunções hepáticas e lesões cutâneas são os principais achados em ruminantes intoxicados (Fagliari et al., 1991a). Os sintomas aparecem após a ingestão das gramíneas portadoras do princípio tóxico - esporidesmina - elaborada pelo fungo (Purchio et al., 1988 ; Fagliari et al., 1991b).

\footnotetext{
* Professores Assistentes Ms do Departamento de Medicina Veterinária - Área de Clínica Médica - UFRPE. Rua Dom Manuel de Medeiros, s/n, Dois Irmãos - Recife - PE CEP 52171-030. e-mail: psoares@ nelore.npde.ufrpe.br

** Professor Adjunto Dr. do Departamento de Medicina Veterinária - Área de Medicina Veterinária Preventiva - UFRPE.
} 
O conhecimento da epidemiologia e aspectos clínicos desta afecção nas diversas regiões do Brasil, principalmente no Nordeste, onde não existe registro na literatura, contribuirá para o controle efetivo desta patologia.

Neste trabalho descreve-se a ocorrência de fotossensibilização em ovinos da raça Santa Inês, no estado de Pernambuco, em condição natural, mantidos em pastagem de Brachiaria decumbens contaminada por Pithomyces chartarum.

\section{Material e métodos}

Em uma propriedade localizada no município de Gravatá-PE, registrou-se a ocorrência de surto de fotossensibilização hepatógena em ovinos. O rebanho era constituído de ovinos da raça Santa Inês, de ambos os sexos, diferentes faixas etárias, agrupados em piquetes constituídos de Brachiaria decumbens.

Investigações a respeito do histórico da doença foram feitas com base em dados de ocorrência, letalidade, faixa etária, sexo, sintomatologia, distribuição geográfica e condições climáticas da propriedade neste período, além de manejo de solo e pastagem, achados de necropsia e exames laboratoriais e de pastagem para pesquisa do fungo em questão. Os animais que apresentavam sintomas foram submetidos a criterioso exame clínico, para registro das lesões.

Para análise laboratorial foi coletado sangue de 12 animais, mediante venopunção jugular, e, posteriormente, separado em dois frascos: um contendo EDTA a 10\%, para realização de hemograma, e outro sem anticoagulante, para obtenção de soro para provas de função hepática e sorologia para leptospirose; a pesquisa de hemoparasitos foi feita através de esfregaço sangüineo. A urina foi coletada por micção espontânea e a urinálise foi realizada utilizando-se fitas reativas Combur 10 Teste. $^{1}$

O hemograma foi realizado de acordo com as técnicas descritas por Jain (1993), onde a contagem total de hemácias e leucócitos foi realizada em Hemocitômetro de Newbauer. A dosagem de hemoglobina foi realizada pelo método da cianometemoglobina, com o auxílio de espectrofotômetro ${ }^{2}$ e Kit para dosagem de hemoglobina. ${ }^{3} \mathrm{O}$ Hematócrito foi efetuado pelo método do microhematócrito, utilizando-se microcentrífuga. ${ }^{4}$ Para a obtenção do volume globular médio (VGM) e concentração de hemoglobina globular média (CHGM), efetuaram-se cálculos apropriados.

A contagem diferencial dos leucócitos foi realizada através da leitura do esfregaço sangüíneo corado pelo método de Leishman. Para avaliação bioquímica-sérica da Bilirrubina Total e frações, gama-glutamil-transferase (GGT) e aspartato amino-transferase (AST), foram utilizados Kits comerciais ${ }^{5}$ e a leitura realizada em espectrofotômetro. ${ }^{6}$

\footnotetext{
1 BOHERINGER - MANHEIM - ALEMANHA

${ }^{2}$ Espectrofotômetro Sectronic Colleman Jr. II

${ }^{3}$ Kit LABTEST

${ }^{4}$ Microcentrífuga HAWKSLEY ENGLAND

${ }^{5}$ Kit LABTEST

${ }^{6}$ Espectrofotômetro CODMAN 195
}

Nos diferentes piquetes foram coletadas, aleatoriamente, amostras de capim para pesquisa dos conídeos do P. chartarum. $O$ exame direto foi feito utilizando-se fita adesiva transparente, sendo esta comprimida sobre a superfície do capim, colocado posteriormente entre lâmina e lamínula, corado pelo azul de Aman e submetido à microscopia (Malavolta et al., 1980).

Dois animais foram conduzidos ao Hospital Veterinário do Departamento de Medicina Veterinária - UFRPE, para acompanhamento intensivo. Um animal foi sacrificado e submetido à necropsia.

\section{Resultados}

Com base nas informações sobre a ocorrência de casos de fotossensibilização hepatógena em ovinos, verificou-se que de um total de 110 animais mantidos em pastagem de $B$. decumbens, 17 apresentaram sintomas compatíveis com a afecção. O surto ocorreu no mês de novembro, período correspondente ao verão no Estado. Neste período, porém, ocorreram chuvas escassas e intercaladas, coincidindo com o início da casuística. Com base no histórico, no mesmo período do ano anterior houve perda de 54 animais e, em propriedade vizinha, 72 animais também vieram a óbito com a mesma sintomatologia, em período de uma semana.

Dos 17 animais que evidenciaram sintomas, cinco morreram e, mediante o quadro clínico, medidas relacionadas ao diagnóstico e controle foram realizadas de imediato. Neste período, encontravam-se agrupados animais de ambos os sexos, porém os machos eram jovens e as fêmeas, na sua maioria, encontravam-se com cria ao pé. A afecção foi observada em animais jovens e adultos, sendo a maioria dos casos em fêmeas adultas.

Na mesma ocasião, um lote de animais machos e adultos foi transferido para outra propriedade, na qual não havia piquete constituído desta pastagem, e nenhum animal havia manifestado quadro clínico semelhante.

Quanto ao manejo de pastagem, verificou-se que esta foi preparada há mais de dois anos, e que um grande número de animais encontrava-se nos piquetes no período em que se identificou o problema. A pastagem encontrava-se úmida e sob intenso pisoteio e mesmo com rotação nos piquetes, havia sobrecarga de animais.

Após avaliação clínica, observou-se: anorexia, apatia, icterícia, edema de pálpebra e orelhas, dermatite na face interna da orelha; opacidade de córnea, vasos episclerais fortemente injetados, necrose e desprendimento de tecido periorbital, além de tumefação e áreas avermelhadas ao redor do olho. Área hepática evidenciando-se aumentada com sensibilidade à percussão e palpação; febre, desidratação e decúbito esternal. $\mathrm{O}$ estado nutricional dos animais era regular.

Quanto aos animais que foram conduzidos ao Hospital Veterinário, verificou-se que o edema de orelha era recidivo, corroborando com as observações feitas pelo proprietário, além de presença de crostas na pálpebra com exsudação ocular. Um dos animais apresentọu significativa depressão do estado sensomotor, sendo sacrificado e submetido à necropsia. Neste, verificou-se icterícia generalizada, fígado amarelado e aumentado de volume, bile verde-escura e com aumento de viscosidade, congestão intestinal, baço com discreto aumento e congestão. 
A sorologia para leptospirose e esfregaço para pesquisa de hemoparasitos foram negativos. Quanto aos parâmetros bioquímico-séricos e hematológicos, os dados obtidos encontram-se nas Tabelas 1 e 2, respectivamente. Na urinálise, verificou-se principalmente hemoglobinúria, variando de moderada a intensa nos animais examinados.

Através da técnica de impressão em fita adesiva, foi possível a identificação de conídeos do referido fungo em grande número das folhas examinadas. Ao exame direto do material, constatou-se a presença de hifas septadas e conídeos pigmentados em forma de barril, com septações transversais e longitudinais.

\section{Discussão}

Em estudos realizados por Malavolta et al. (1980) com o objetivo de identificar problemas relacionados com o $P$. chartarum, constatou-se ocorrência em nove Estados e Distrito Federal, não tendo registro da ocorrência de intoxicação por este fungo em ovinos na região Nordeste do Brasil.

Segundo Schenk, Schenk (1983), as espécies animais que, em condição natural, têm sido observadas com fotossensibilização são a bovina, bubalina e ovina. Avaliando os parâmetros zootécnicos e os registros, não se encontraram dados na literatura que caracterizassem a clínica de fotossensibilização hepatógena em ovinos da raça Santa Inês, a qual apresenta pele e pêlos de cor escura, diferenciando dos ovinos citados na literatura com eczema facial.

Franzolin Neto (1985), Purchio et al. (1988) e Coyne e Oehme (1993) reportaram que tal processo ocorre com o aparecimento de lesões em pele restritas às áreas não pigmentadas e expostas aos raios solares. Porém Coyne e Oehme (1993) acrescentam que os animais sensivelmente afetados podem apresentar envolvimento das regiões pigmentadas da pele. Corroborando-se, portanto, as observações feitas neste estudo, onde se verificaram lesões típicas de fotossensibilização na cabeça, caracterizando-se, assim, a sintomatologia e patologia para esta raça, com importância na epidemiologia e controle desta afecção.

No Brasil a prevalência mais acentuada de intoxicação por $P$. chartarum foi verificada em espécies da família Graminaceae ( $B$. decumbens, $B$. radicans e $B$. brizantha) (Döbereiner et al., 1976 ; Tokarnia et al., 1979), cuja espécie de forrageira encontrada nos piquetes onde se registrou a ocorrência foi a $B$. decumbens, planta bastante difundida no

Tabela 1: Apresentação da estatística descritiva de média (X) de variáveis bioquímica-sérica de ovinos intoxicados naturalmente por $P$. chartarum em pastagem de $B$. decumbens

\begin{tabular}{cccccc}
\hline \multirow{2}{*}{ Animais } & \multicolumn{5}{c}{ Avaliação bioquímica sérica } \\
\cline { 2 - 6 } & $\begin{array}{c}\text { B .total } \\
(\mathrm{mg} / \mathrm{dl})\end{array}$ & $\begin{array}{c}\text { B. direta } \\
(\mathrm{mg} / \mathrm{dl})\end{array}$ & $\begin{array}{c}\text { B. indireta } \\
(\mathrm{mg} / \mathrm{dl})\end{array}$ & $\begin{array}{c}\text { AST } \\
(\mathrm{Ul} / \mathrm{L})\end{array}$ & $\begin{array}{c}\text { GGT } \\
(\mathrm{UI} / \mathrm{L})\end{array}$ \\
\hline 06 & 0,7 & 0,3 & 0,4 & 358,6 & 93,0 \\
07 & 0,6 & 0,4 & 0,2 & 361,0 & 142,7 \\
08 & 0,6 & 0,4 & 0,2 & 266,1 & 118,6 \\
10 & 0,3 & 0,2 & 0,1 & 164,4 & 90,6 \\
Valores de & 0,1 a 0,42 & 0,0 a 0,27 & 0,0 a 0,12 & 264 a 350 & 20 a 52 \\
Referência* & & & & &
\end{tabular}

KANECO (1989).
Tabela 2: Apresentação de estatística descritiva de variáveis hematológicas de ovinos $(n=12)$ intoxicados naturalmente por $P$. chartarum em pastagem de $B$. decumbens.

\begin{tabular}{lccccc}
\hline \multirow{2}{*}{ Variáveis } & & & \multicolumn{3}{c}{$\mathrm{IC}$} \\
\cline { 4 - 5 } & $\mathrm{x}$ & $\mathrm{s}$ & $\mathrm{LI}$ & $\mathrm{LS}$ & $\mathrm{MD}$ \\
\hline Eritrócitos $\left(\times 10^{6} / \mathrm{mm}^{3}\right)$ & 10,86 & 1,62 & 9,94 & 11,78 & 10,41 \\
Hemoglobina $(\mathrm{g} / \mathrm{dl})$ & 7,58 & 1,36 & 6,81 & 8,35 & 8,10 \\
Hematócrito $(\%)$ & 41,33 & 4,18 & 38,97 & 43,70 & 41,50 \\
VGM (fl) & 38,88 & 5,33 & 35,87 & 41,90 & 38,30 \\
CHGM (\%) & 18,40 & 3,33 & 16,52 & 20,28 & 19,45 \\
Leucócitos $\left(\times 10^{6} / \mathrm{mm}^{3}\right)$ & 11,26 & 4,57 & 8,67 & 13,84 & 9,95 \\
Segmentados $(\%)$ & 59,33 & 15,39 & 50,62 & 68,04 & 58,50 \\
Linfócitos $(\%)$ & 37,66 & 14,32 & 29,56 & 45,76 & 39,00 \\
Eosinófilos $(\%)$ & 1,83 & 1,69 & 0,41 & 1,92 & 2,00 \\
Monócitos $(\%)$ & 1,16 & 1,34 & 0,87 & 2,79 & 1,00 \\
\hline
\end{tabular}

X: Média, s: Desvio-padrão, IC: Intervalo de confiança, LI: Limite-inferior, LS: Limite superior, MD: Mediana.

Estado de Pernambuco. Coyne e Oehme (1993) citaram que o eczema facial está associado à pastagem em locais de grama ressecada ou legumes mofados, infectados por fungos saprófitas, como o $P$. chartarum registrado no exame da pastagem em questão.

Foi observado na Região de São Marcos, Estado de São Paulo, por Purchio et al. (1988), que a freqüência da contaminação aumenta no final do verão e início de outono quando ocorre elevação da temperatura e umidade relativa do ar, além de sucessão de pequenas precipitações pluviométricas (DiMenna, Bailey, 1973 ; Nazário, 1979 ; Fagliari et al., 1993). Isto se deve ao fato de que, em épocas do ano com baixas precipitações pluviométricas, as folhas caem e, quando o manejo da pastagem não é correto, há sobras de pasto que se depositam no solo, produzindo microclima favorável à esporulação do referido fungo (Fagliari et al., 1993). Tais condições foram observadas na propriedade em estudo.

Além dos aspectos climáticos, a predisposição individual e o estado nutricional são considerados fatores relevantes para o desencadeamento da doença (Purchio et al., 1988). Relacionando-se ao estado nutricional, verificou-se compatibilidade com a falha de manejo, pois não havia suplementação protéica e mineral, estando os animais dispostos em período de menor oferta de alimento na época da ocorrência.

Com relação à faixa etária, a maioria dos animais envolvidos era adulta, e tais observações estão de acordo com Döbereiner et al. (1976) e Tokarnia et al. (1979). Quanto aos neonatos, em que se observaram doentes e casos de óbitos, com registro de ocorrência com as mesmas características no ano anterior, inclusive com maior número de óbitos, estes se mantiveram enfermos juntamente com as matrizes. Conforme Fagliari et al. (1983) e Fagliari et al. (1993), que afirmaram haver possibilidades de transferência da toxina pelo leite, o que poderia proporcionar alteração no estado de saúde dos lactantes, verificou-se, por meio do respectivo inquérito, a hipótese da ocorrência por este fator, além de outros fatores inerentes ao meio, associados ao estado geral das matrizes e dos neonatos. Afirma-se, portanto, a necessidade de inquéritos mais aprofundados para confirmar esta hipótese. 
Os dados de sintomatologia, observados nesta ocorrência, evidenciaram lesões na cabeça, principalmente na região periorbital e orelhas. Quanto ao eczema facial, os dados da clínica são de prurido nasal e ao redor dos olhos, edema facial e de orelhas, mucosas ictéricas, perda de peso e prostração (Purchio et al., 1988), cujos sintomas se diferenciam dos observados nestes animais, visto que o eczema facial apresenta uma sintomatologia bem mais evidente e ampla na cabeça.

Temperine e Barros (1977) verificaram que carneiros, recebendo $1 \mathrm{mg} / \mathrm{kg}$ de esporidesmina, apresentavam após intervalo de tempo, níveis desta micotoxina em líquidos orgânicos. Observaram a rapidez com que a esporidesmina aparece na bile, ou seja, cerca de 10 minutos após a administração, mostrando que a sua absorção é rápida. Desta forma, confirma-se a hipótese de se observar grande número de óbitos em reduzido intervalo de tempo, quando se verificou a clínica nos animais em questão.

O eczema facial constitui-se primariamente dos danos hepáticos, que podem produzir secundariamente sintomas de fotossensibilização, pelos quais a doença é reconhecida no campo (Temperine, Barros, 1977 ; Coetzer et al., 1983). A icterícia observada em todos os animais com sintomatologia não é, segundo Hore (1960), observação constante, variando consideravelmente em diferentes graus, sendo observada nos tecidos subcutâneos e adiposo distribuídos por todo o corpo, o que foi observado nestes animais.

Em relação ao eritrograma, verificou-se que o valor médio do CHGM obtido a partir das 12 amostras analisadas encontrava-se abaixo do valor mínimo segundo Blood e Radostits (1991) e Jain (1993), caracterizando uma anemia do tipo normocítica e hipocrômica. De acordo com esses autores, esta pode ser um tipo de alteração relacionada a uma deficiência nutricional ocasionada por uma carência de cobre, por parasitismo hematófago ou perda crônica de sangue. Na propriedade em questão os animais não apresentavam histórico de vermifugação nos últimos meses além de uma hemoglobinúria, comprovada pela urinálise, nos animais que apresentaram sintomas. Quanto ao leucograma, observouse uma inversão na relação neutrófilo-linfócito, que pode ser interpretado como reflexo da hiperatividade adrenocortical conseqüente à condição de estresse do quadro clínico primário ou associado ao processo inflamatório agudo (Jain, 1993).

AST é indicativo inespecífico da necrose hepática e tende a aumentar gradualmente, permanecendo elevada por vários dias (Carlson, 1993). De acordo com a Tabela 1, verificou-se

\section{Referências bibliográficas}

BAGLEY, C. V. 1983. Photosensitization associated with expusure of cattle to moldy straw. J Am Vet Med Assoc, v. 183, n. 7, p. 802-803, 193.

BLOOD D. C., RADOSTITS, O. M. Clínica Veterinária. 7. ed., Rio de Janeiro : Guanabara Koogan, 1991.

CAMARGO, W.V.A., NAZARIO, W., FERNANDES, N. S., AMARAL, R. E. M. Fotossensibilização em bovinos de corte. Provável participação do fungo Pithomyces chartarum na etiologia do processo. Biológico n. 42 , p. 259-261, 1976. que dois dos quatro animais avaliados apresentaram elevações discretas desta enzima, podendo estar relacionada ao dano hepático recente. As alterações da gama GT são compatíveis com o dano hepático grave e insuficiência hepática(Carlson, 1993), o qual foi observado em todos os animais. Em relação às bilirrubinas séricas, constatou-se um discreto aumento da BT em três dos quatro animais avaliados, sendo que em dois deles foi observada uma elevação da BD e em um animal uma elevação da BI. Kaneko (1989) comenta que as bilirrubinas séricas estão discretamente aumentadas nas doenças hepáticas graves e difusas em ruminantes, com aumento marcado em casos terminais. Carlson (1993) relaciona como causa comum do aumento da bilirrubina de reação indireta uma insuficiência hepática resultante na absorção/conjugação prejudicadas da bilirrubina e uma insuficiência hepática aguda causada por necrose hepática, resultando em aumento de moderada a marcante da BD e BI. Nos animais em questão foi observado um aumento leve da bilirrubina total, sugerindo uma lesão hepática ativa e grave.

Com relação ao exame do material coletado na propriedade para identificação do fungo, a técnica empregada está de acordo com Malavolta et al. (1980), a qual mostrou ser prática e eficiente na identificação do referido fungo. De acordo com Temperine e Barros (1977), não há risco de eczema facial no campo, a menos que o número de esporos nas pastagens esteja alta, acima de $10^{5} \mathrm{~g}$ de peso da pastagem. Tal técnica de quantificação não foi realizada, porém pressupõe-se, com base nos dados obtidos, que este limiar foi alcançado.

No exame post-mortem, salientavam-se lesões de pele, aumento de fígado, vesícula biliar repleta e icterícia generalizada (Nazário, 1979 ; Schenk, Schenk, 1983), com evidência dos ductos biliares (Dobereiner et al., 1976). Com relação às observações feitas neste estudo, verificou-se concordância com os autores citados, cujos achados necroscópicos são determinantes de uma hepatopatia tóxica.

\section{Conclusões}

Ovinos da raça Santa Inês, com pele e pêlos pretos, ingerindo pastagem de $B$. decumbens contaminada por $P$. chartarum, podem ser acometidos de lesões de fotossensibilização hepatógena com caracterização clínica de edema e necrose tissular nas orelhas e região periorbital; apresentam alterações hematológicas, enzimas hepáticas, urinálise e lesões macroscópicas de órgãos abdominais, e estas são relevantes no auxílio diagnóstico desta afecção, além dos dados referentes às condições de manejo.

CARLSON, G. P. Testes de Química Clínica, In: Tratado de Medicina Interna de Grandes Animais: moléstias de equinos, bovinos, ovinos e caprinos São Paulo:Manole. p. 395-423. 1993

COETZER, J. A. W., KELLERMAN, T. S., SADLER, W., BATH G. F. Photossensitivity in South Africa. V. A comparative study of the pathology of the ovine hepatogenous photosensitivity diseases, facial eczema and special geeldikkop (Tribulosis ovis), with special reference to their athogenesis. J Vet Res n. 50, p. 59-71, 1983.

COYNE, C.P., OEHME, F. W. Toxinas de ocorrência natural. In: Tratado de Medicina Interna de Grandes Animais: moléstias de equinos, bovinos, ovinos e caprinos. p. 1639-1660. São Paulo: Manole, 1993. 
DIMENNA, M. E., BAILEY, J. R. Pithomyces chartarum spore counts in pasture. NZJ of Agric Res n. 16, p. 343-351, 1973.

DÖBEREINER, J., TOKARNIA, C. H., MONTEIRO, M. C. C., CRUZ, L. C. H., CARVALHO, E. G., PRIMO, A. T. Intoxicação de bovinos e ovinos em pasto de Brachiaria decumbens contaminados por Pithomyces chartarum. Pesq Agropec Bras, n. 11, p. 87-94, 1976.

FAGLIARI, J. J., PASSIPIERI, M., OLIVEIRA, J. A. Sintomas de fotossensibilização em bezerros alimentados com leite materno. Arq Bras Med Vet Zootv. 35, n. 4, p. 479-484. 1983.

, KUCHEMBUCK, M. R. G., CURI, P. R., PASSIPIERI, M. Aspectos epidemiológicos da intoxicação natural de bovinos pela micotoxina esporodesmina. Ciência Veterinária v. 5, n. 1, p. 1-5, 1991 a.

KUCHEMBUCK, M. R. G., CURI, P. R., OKUDA, H. T. Aspectos clínicos da intoxicação natural de bovinos pela micotoxina esporodesmina. Ciência Veterináriav. 5, n. 2, p. 1-3, 1991 b.

MASSIPIERI, M., KUCHEMBUCK, M. R. G., CURI, P. R. Intoxicação natural de bovinos pela micotoxina esporidesmina. II. Aspectos clínicos. Arq Bras Med Vet Zoot v. 45, n. 3, p. 275-282, 1993.

FRANZOLIN NETO, R. Fotossensibilização em animais sob pastejo em gramíneas tropicais. Comun Cient Fac Med Vet Zootec Univ S Paulo, v. 9, n. 1, p. 19-26, 1985.

HANSEN, D. E., MCCOY, R. D., HEDSTROM, O. R., SNYDER, S. P., BALLERSTEDT, P. B. Photosensitization associated with expousure to Pithomyces chartarum in lambs. J Am Vet Med Assoc. v. 204, n. 10, p. 1668-1671, 1994.

HORE, D. E. Facial eczema. Australian Vet Jourp. 172-176, 1960.

JAIN, N. C. Essentials of Veterinary Hematology, Philadelphia : Lea \& Febiger, 417 p., 1993.
KANEKO, J. J. Clinical Biochemistry of Domestic Animals, California: Academic Press Inc, 932 p.1989.

MALAVOLTA, V. M. A., AMARAL, R. E. M., PIPINSKAS, O. M.. Plantas hospedeiras e distribuição geográfica do fungo Pithomyces chartarum (BERK \& CURT) MB ELLIS no Brasil. Biológico, v. 46 n. $3 /$ 4, p. 59-76, 1980.

MUCHIRI, D. J., BRIDGES, C. H., UECKERT, D. N., BAILEY, E. M. Photosensitization of sheep on kleingrass pasture. J Am Vet Med Assocv. 177, n. 4, p. 353-354, 1980.

NAZARIO, W. Revisão das micotoxicoses de interesse Médico e Veterinário. Biológico, v. 45, n. 7/8, p. 113-122, 1979.

NOBRE, D., ANDRADE, S. O. Relação entre fotossensibilização em bovinos jovens e a gramínea Brachiaria decumbens Stapf. Biológico, v. 42, p. 249-258, 1976.

PURCHIOA., CORREAB, GALHAR, D. M., PELICCI, P. Ocorrência de surto de eczema facial em ovinos na região de São Manuel, Estado de São Paulo. Rev FacMed Vet Zootec S Paulo, v. 25, n. 1, p. 135-141, 1988.

RICHARD, J. L. Mycotoxin photosensitivity. J Am Vet Med Assoc, v. 163, n. 11, p. 1298-1301, 1973.

SCHENK, M. A. M., SCHENK, J. A. P. Aspectos gerais da fotossensibilização hepatógena em bovinos. EMBRAPA-CNPGC, Comunicado técnico, n. 19, p. 1-7, 1983.

TEMPERINE, J. A., BARROS, M. A. Revisão sobre os aspectos químicos e físicos ligados aos fenômenos de fotossensibilização e efeitos biológicos da esporidesmina obtida do fungo Pithomyces chartaraum (BERK \& CURT) M.B. ELLIS. Biológico, n. 43, p. 103-110. 1977.

TOKARNIA, C. H., DÖBEREINER, J., SILVA, M. F. Plantas Tóxicas da Amazônia a Bovinos e Outros Herbívoros. Instituto de Pesquisa da Amazônia, Manaus. 95 p., 1979. 